\title{
RESPUESTAS DIFERENCIALES PARA RENDIMIENTO DE HÍBRIDOS DE MAIZ EVALUADOS EN AMBIENTES CONTRASTANTES DE LATINOAMÉRICA PCCMCA 1990*
}

\author{
Hugo Córdova**
}

\begin{abstract}
RESUMEN
Se evaluó el ensayo uniforme del PCCMCA que involucró 36 hibrídos de maíz en 20 ambientes contrastantes de Latinoamérica. Los resultados de 1990 comprueban la estabilidad de la respuesta de algunos híbridos que durante tres años a través de 51 localidades han permanecido en los primeros lugares: HB-85, HB-83 y XH-53 con rendimientos hasta de $6,000 \mathrm{~kg} / \mathrm{ha}$ y $27 \%$ sobre el testigo. Sus variables de estabilidad ( $\mathrm{B}=1$ y Sdi ${ }^{2}=0$ ) confirman lo acertado de esta respuesta. Nuevos híbridos de Compañías Privadas y Programas Nacionales demuestran un continuo progreso en el mejoramiento.
\end{abstract}

\begin{abstract}
The uniform assay of the PCCMCA involves the evaluation of 30 coro hybrids in 20 contrasting environments in Latin America. The results from 1990 prove the consistency of response of come coro hybrids which have remaines in the first places during three years through 51 localities: HB-85, HB-83 and XH-53, with yields up to 6000 $\mathrm{kg} / \mathrm{ha}(27 \%$ over the control). The stability parameters confirm the fitness of this response. New hybrids from private companies and national programmes show a continuous progress in plant breeding.
\end{abstract}

\section{INTRODUCCION}

El mejoramiento para resistencia a factores adversos bióticos y abióticos en maíz han dado como resultado el desarrollo de híbridos más estables adaptados a la mayoría de condiciones de producción, así mismo menciona que los modelos de Eberhart y Russell y AMMI coinciden en el $40 \%$ de los casos para identificar híbridos estables y rendidores. El rendimiento y la estabilidad del comportamiento deben considerarse simultáneamente, para reducir los efectos de la interacción GxA y para una selección más precisa y refinada, Kang (1990).

Una evaluación realista del comportamiento de cultivares adaptados a ambientes pobres y ricos, debe involucrar localidades cuya magnitud de la incidencia de factores adversos bióticos y abióticos contribuya a reducir la producción. La aplicación de modelos donde se estiman parámetros de estabilidad que indentifican el comportamiento de los cultivares a través de diversos ambientes, contribuyen a la selección apropiada de los genotipos, Córdova (1989).

El mejoramiento para adaptación amplia puede proveer la identificación de genotipos cuyo

\footnotetext{
* Trabajo presentado en la XXXVII Reunión Anual del PCCMCA, Panamá. 1991.

** Representante Regional de Maíz de CIMMYT, para Centroamérica y el Caribe
}

comportamiento sea superior en ambientes pobres y ricos, estos ambientes pueden estar caracterizados por condiciones de estrés. Un cultivar estable bajo estas circunstancias poseerá una baja interacción genotipo por ambiente, un coeficiente de regresión cercano a 1,0 y un alto rendimiento. La importancia que tienen los factores responsables del rendimiento a través de ambientes contrastantes, y la fracción de esos factores específicos es muy relevante para entender la naturaleza de la estabilidad del rendimiento y puede abrir un camino para el desarrollo de criterios de selección adicionales al rendimiento, Blum (1988).

Con base en modelos de regresión e índices ambientales se han establecido parámetros de estabilidad para determinar el comportamiento de cultivares a través de una serie de ambientes contrastantes. Genotipos con una media de rendimiento alta, $\mathrm{B}=1$ y $\mathrm{Sdi}^{2}=0$ son definidos como estables en la concepción del último de los autores. Durante los últimos 10 años estos modelos han sido utilizados ampliamente en Centroamérica y El Caribe, Córdova et al (1988), Yates y Cochram (citados por Blum 1988) Finlay y Wilkinson (1963);Eberhart y Ruseell (1966). 
El modelo AMMI (efectos principales aditivos e interacción multiplicativas), es el modelo a escoger cuando existen datos de experimentos que muestran significancia en los efectos principales y sus interacciones, AMMI integra algunos modelos estadísticos comúnmente aplicados a series de ensayos de rendimiento, esta integración incluye el análisis de varianza (ANOVA cuyo modelo es aditivo, el análisis de componentes principal (PCA) el cual es multiplicativo y el modelo de regresión lineal de Finlay-Wilkinson, Gauch y Zobel (1989).

El modelo AMMI ha demostrado eficiencia por las razones siguientes: 1 . Es más fácil el entendimiento de la interacción genotipo-ambiente al utilizar los Scores PCA presentados gráficamente, Kempton (1984) y Zobel et al. (1988). 2. Predicción de estimaciones del rendimiento más precisas al descartar en residual con mucho "ruido". 3. La mayor precisión se traduce en nuevas opciones para crear diseños experimentales con menos repeticiones y mayor número de tratamientos. 4. Mayor precisión mejora el éxito en seleccionar el material realmente superior (3 años sin usar AMMI equivalen a dos años usando AMMI). S.El residual del AMMI puede revelar hetereogenidad en los experimentos en el campo. 6. El mejor entendimiento de las interacciones y la mayor precisión en las estimaciones del rendimiento hacen posible las recomendaciones de variedades más confiables y mayor progreso en el programa de mejoramiento, Gauch y Zobel (1989).

La estabilidad de una cruza doble proviene de la mezcla de genotipos,pero Eberhart y Russell (1966) indican que también parece que está bajo control genético o sea que ciertos genotipos pueden mostrar mayor estabilidad que otros, de manera que pueden obtenerse cruzas simples, genéticamente estables de mayor rendimiento que las cruzas dobles. En su investigación encontraron cruzas simples tan estables como cualquier cruza doble, sugiriendo que las cruzas simples difieren en su habilidad de respuestas a condiciones ambientales más favorables; la suma de cuadrados de desviaciones de regresión parece ser el parámetro más importante y que es probable que estén involucrados en esa estabilidad todos los tipos de acción genética.

Sprague y Jenkins (1943) citados por Córdova (1978) y Allard y Bradshaw (1967), coinciden en que la mayor diversidad genética (cruzas simples en maíz, por ejemplo) dota a las poblaciones de mayor estabilidad, haciéndolas idóneas para utilizarse también en ambientes desfavorables.
En la estimación de parámetros de estabilidad en variedades de maíz (Carballo y Márquez, 1970), hacen notar que en el grupo de variedades de alto rendimiento, los coeficientes $\mathrm{b}$ no difirieron mucho de 1 o son inferiores a este valor. Mencionan que la tendencia general fue la asociación de altos rendimientos con altos valores de B y la asociación negativa de rendimiento y de B con $\mathrm{Sdi}^{2}$.

Con base en el modelo de Eberhart y Russell (1966), Dávila, Córdova y Poey (1978), estimaron los parámetros de estabilidad para identificar germoplasma criollo utilizable en el Programa de Mejoramiento del Altiplano, alto y medio. Los autores concluyen que dentro del germoplasma criollo existen variedades con alto potencial de rendimiento y estabilidad mostrada a través de nueve localidades del altiplano medio de Guatemala; a la vez encontraron que altos rendimientos están positivamente correlacionados a coeficientes de regresión y desviaciones de regresión ( $\mathrm{r}=0,99$ y 0,66 , respectivamente).

Este trabajo tiene los siguientes objetivos:

a. Determinar la adaptación de los híbridos de maíz desarrollados por los Programas Nacionales y Compañías Privadas en las diferentes regiones maiceras de Centroamérica y El Caribe.

b. Estimar parámetros de estabilidad que permiten describir los genotipos de acuerdo a su respuesta a través de ambientes contrastantes.

c. Realizar un análisis combinado de años y localidades a través de un modelo comprensible que permita inferencias acertadas.

d. Establecer un mecanismo oficial en el cual los Programas Nacionales puedan basar sus decisiones en cuanto a la selección del germoplasma adecuado a las circunstancias de cada país, de tal manera que la información de varias localidades y años analizada en forma combinada genere recomendaciones más confiables.

\section{MATERIALES Y METODOS}

En el presente estudio se evaluaron 36 híbridos desarrollados por los Programas Nacionales, compañías privadas nacionales y compañías privadas internacionales 
que operan en la región de Centroamérica. Los 36 híbridos fueron evaluados en ensayos uniformes en 22 localidades de América Central, El Caribe, México, Estados Unidos y Venezuela, bajo un diseño de látice simple cuadrado $6 \times 6$ con cuatro repeticiones por localidad, (Cuadro 1).

Cuadro 1. Híbridos de maíz evaluados en el ensayo del PCCMCA 1990.

\begin{tabular}{|c|c|c|c|c|}
\hline ENTRADA & \multirow{2}{*}{$\begin{array}{l}\text { NOMBRE } \\
\text { H3 } 501\end{array}$} & \multirow{2}{*}{$\begin{array}{l}\text { INSTITUCION } \\
\text { C. BURKARD }\end{array}$} & \multirow{2}{*}{$\frac{\text { PAIS }}{\text { Guatemala }}$} & \multirow{2}{*}{$\frac{\text { COLOR }}{\text { Blanco }}$} \\
\hline 1 & & & & \\
\hline 2 & CB $x$ HS 503 & C. BURKARD & Guatemala & Blanco \\
\hline 3 & CB $\times$ HS 505 & C. BURKARD & Guatemala & Blanco \\
\hline 4 & CB $\times$ HS 507 & C. BURKARD & Guatemala & Blanco \\
\hline 5 & $\mathrm{HR}-12$ & SEMINAL & Guatemala & Amarillo \\
\hline 6 & $\mathrm{HR}-17$ & SEMINAL & Guatemala & Blanco \\
\hline 7 & TACSA H203 & TACSA & México & Amarillo \\
\hline 8 & EXP. 114 & ICTA & Guatemala & Amarillo \\
\hline 9 & GB-39 $x$ GB-41 & ICTA & Guatemala & Blanco \\
\hline 10 & MAX307 & AGRIDEC & U.S.A. & Blanco \\
\hline 11 & MAX311 & AGRIDEC & U.S.A. & Blanco \\
\hline 12 & MAX319 & AGRIDEC & U.S.A. & Blanco \\
\hline 13 & PIONNER WF $6 \times \mathrm{XH} 53$ & PIONNER & U.S.A. & Amarillo \\
\hline 14 & PIONNER WF $6 \times$ CJ66 & PIONNER & U.S.A. & Amarillo \\
\hline 15 & $\mathrm{H}-30$ & DIA & Honduras & Blanco \\
\hline 16 & $\mathrm{H}-33$ & DIA & Honduras & Blanco \\
\hline 17 & $\mathrm{H}-29$ & DIA & Honduras & Blanco \\
\hline 18 & $\mathrm{H}-5$ & CENTA & El Salvador & Blanco \\
\hline 19 & $H-53$ & CENTA & E1 Salvador & Blanco \\
\hline 20 & $\mathrm{H}-56$ & CENTA & E1 Salvador & Blanco \\
\hline 21 & $\mathrm{HB}-83$ & ICTA & Guatemala & Blanco \\
\hline 22 & $\mathrm{HC}-43$ & UCR & Costa Rica & Blanco \\
\hline 23 & GB-35xGB4 1 & ICTA & Guatemala & Blanco \\
\hline 24 & $\mathrm{HA}-46$ & ICTA & Guatemala & Amarillo \\
\hline 25 & $C-125 \mathrm{~W}$ & CARGIL & México & Blanco \\
\hline 26 & $C-343$ & CARGIL & México & Blanco \\
\hline 27 & $C-385$ & CARGIL & México & Blanco \\
\hline 28 & $C-701$ & CARGIL & México & Amarillo \\
\hline 29 & GB-43 $x$ GB -45 & ICTA & Guatemala & Blanco \\
\hline 30 & $\mathrm{HB}-85$ & ICTA & Guatemala & Blanco \\
\hline 31 & EXP. HQPM1 & ICTA & Guatemala & Amarillo \\
\hline 32 & EXP. HQPM2 & ICTA & Guatemala & Amarillo \\
\hline 33 & CB-XHS- 502 & C. BURKARD & Guatemala & Amarillo \\
\hline 34 & CB-XGS-509 & C. BURKARD & Guatemala & Blanco \\
\hline 35 & CB-XHS-511 & C. BURKARD & Guatemala & Blanco \\
\hline 36 & CB-XHS- 513 & C. BURKARD & Guatemala & Blanco \\
\hline
\end{tabular}

La parcela experimental constó de 4 surcos de $5,5 \mathrm{~m}$ de largo con una parcela útil de $8,25 \mathrm{~m}^{2}$, densidad de 53,000 plantas ha. La información climática de los sitios experimentales se encuentra compilada en la base de datos de los ensayos del PCCMCA, lo cual indica que los 22 experimentos fueron establecidos bajo condiciones de mega ambiente tropical con temperaturas promedio entre $22^{\circ}$ y $28^{\circ} \mathrm{C}$ precipitación entre 550 y $1100 \mathrm{~mm}$ durante el ciclo de cultivos y altitud entre 0 y $1000 \mathrm{msnm}$. Se aplicó entre 100 y $120 \mathrm{~kg} / \mathrm{ha}$ de $\mathrm{N}$ y $50 \mathrm{~kg} / \mathrm{ha}$ de $\mathrm{P}_{2} 0_{5}$.

Las variables estudiadas fueron: rendimiento, días a flor, altura de planta y mazorca, enfermedades de importancia económica, pudrición de mazorca, cobertura de mazorca, acame y profilicidad.

La preparación de los ensayos de 1990 correspondió a Guatemala, los ensayos fueron enviados a 26 localidades, de las cuales al 15 de febrero de 1990 se recibieron 22 libros de campo, o sea 86\% de recuperación lo cual se considera sobresaliente. En el análisis de estabilidad se utilizaron datos de 20 ensayos. Los datos de rendimiento se expresan en t/ha de grano al $15 \%$ de humedad. Se seleccionaron además los seis mejores híbridos durante el período 1988-1990 incluyendo al testigo H-5 que involucra a 51 localidades y 204 repeticiones.

\section{Análisis estadístico}

Se realizó un análisis de varianza por localidad bajo el modelo de látice para rendimiento, altura de mazorca, días a flor, mazorcas descubiertas y podridas y \% de prolificidad.

Yijq $=\mathrm{U}+\mathrm{i}+\mathrm{Bij}+\mathrm{Tq}+\mathrm{eijq}$

Yijq = Efecto del q-ésimo tratamiento en el j-ésimo bloque dentro de la i-ésima repetición.

$\mu=$ Efecto de la media

$\mathrm{i}=$ Efecto de la repetición

$\mathrm{Bij}=$ Efecto del bloque incompleto

$\mathrm{Tq}=$ Efecto del tratamiento

Eijq $=$ Efecto del error

Las comparaciones de medias se realizaron por la prueba de Tukey (DMSH).

\section{Análisis de estabilidad}

El análisis combinado de estabilidad para 1990, se realizó bajo el modelo de Eberhart y Russell (1966).

$\mathrm{Yij}=\mathrm{Mi}+\mathrm{Bi}+\mathrm{Ij}+\mathrm{Sij}$

Yij = Media varietal de la i-ésima variedad en el j-ésimo ambiente $(1=1,2 \ldots \mathrm{V} ; \mathrm{J}=1,2,3 \ldots \mathrm{N})$.

$\mu=$ La media de la i-ésima variedad a través de todos los ambientes.

$\mathrm{Bi}=$ Coeficiente de regresión que mide la respuesta de la variedad $\mathrm{i}$ en varios ambientes. $\mathrm{Ij}=$ Indice ambiental obtenido como el promedio de todas las variedades en el j-ésimo ambiente menos la media general.

Sij = Desviación de regresión de la variedad i en el ambiente j. 
Para establecer la relación existente entre la descripción de estabilidad de Eberhart y Russell, se analizaron los resultados por el modelo AMMI donde:

yge $=\mu+$ Óg $+\beta e+$ õnyn rgn + ópge $=$ Rendimiento promedio de un genotipo $g$ en ambiente e.

$\mu=$ Es la media general

Óg = Desviaciones de las medias de los genotipos.

$\mathrm{Be}=$ Desviaciones de las medias de ambiente.

$\mathrm{N}=$ Número de PCA retenidos en el modelo.

$\mathrm{y}=$ Valor singular para el PCA.

rgn $=$ Valores de vectores de los genotipos para cada ambiente PCA.

$\tilde{\mathrm{o}}=$ Valores de los sectores para cada ambiente (PCA).

Pge $=$ Es el residual.

\section{Análisis de correlaciones simples}

Para estudiar el grado de asociaciones entre rendimiento y características agronómicas de baja heredabilidad como mala abertura y pudrición de mazorca, se estimaron coeficientes de correlación simple.

\section{RESULTADOS Y DISCUSIÓN}

Algunos de los cultivares evaluados en veinte localidades de Centroamérica, El Caribe, Venezuela, México y Estados Unidos, mostraron comportamiento relativamente diferentes a los ambientes contrastantes donde fueron evaluados Córdova (1978),Córdova et al. 1988, Córdova 1989.

En el Cuadro 2, se presentan los valores estimados en el análisis de varianza para rendimiento por localidad. El experimento que obtuvo el valor más bajo de coeficiente de variación C.V. $(5,0)$ fue la localidad de San Jerónimo, Guatemala y el valor más alto $(19,2)$ para la localidad de Guadalajara, México.

Los índices ambientales indican ambientes constrastantes donde fueron evaluados los 36 cultivares involucrados en el presente trabajo (Cuadro 3).

Cuadro 2. Estimadores en el análisis de varianza para rendimiento de 36 híbridos de evaluados en 20 localidades de Norte y Centroamerica, El Caribe y Venezuela. PCCMCA, 1.

\begin{tabular}{|c|c|c|c|c|c|c|c|}
\hline País & Localidad & Código & Media & C.V. & $\mathrm{Fa}=0 ; \mathrm{a}=, 05$ & MDS & $\mathrm{DEE}$ \\
\hline Guatemala & Tiquizate & 9001 & 3,18 & 12,8 & $\dot{*} *$ & 0,813 & 0,575 \\
\hline Guatemala & Cuyuta & 9002 & 3,56 & 11,9 & $\dot{*} *$ & 0,790 & 0,559 \\
\hline Guatemala & La Máquina & 9003 & 5,53 & 7,1 & $x *$ & 0,730 & 0,516 \\
\hline Guatemala & San Jeronimo & 9004 & 8,29 & 5,0 & $\dot{x}$ & 0,786 & 0,558 \\
\hline Honduras & Danlí & 9005 & 4,95 & 17,1 & $* *$ & 1,610 & 1,140 \\
\hline Honduras & Omonita & 9006 & 6,77 & 7,7 & $* *$ & 0,963 & 0,681 \\
\hline Honduras & Zamorano & 9007 & 5,33 & 9,4 & $* *$ & 0,946 & 0,669 \\
\hline Honduras & La Ceiba & 9008 & 4,24 & 19,8 & $\dot{x} \dot{x}$ & 1,580 & 1,11 \\
\hline El Salvador & Santa Cruz & 9009 & 5,19 & 8,7 & $* *$ & 0,587 & 0,614 \\
\hline E1 Salvador & San Andres & 9010 & 4,70 & 6,9 & $* *$ & 0,628 & 0,444 \\
\hline Nicaragua & Jalapa & 9011 & 6,42 & 9,1 & $* *$ & 1,140 & 0,807 \\
\hline Nicaragua & San Cristobal & 9012 & 2,21 & 13,6 & ix & 0,590 & 0,421 \\
\hline Costa Rica & Alajuela & 9013 & 8,24 & 6,4 & $x *$ & 0,987 & 0,697 \\
\hline Panamá & La Honda & 9015 & 5,76 & 7,2 & $* *$ & 0,591 & 0,418 \\
\hline Panamá & Parita & 9016 & 4,90 & 7,2 & $* *$ & 0,651 & 0,460 \\
\hline Rep. Dominicana & San Cristobal & 9017 & 3,72 & 11,7 & $* *$ & 0,800 & 0,566 \\
\hline México & Tapachula & 9020 & 6,30 & 6,9 & $* *$ & 0,837 & 0,592 \\
\hline México & Guadalajara & 9021 & 6,85 & 19,2 & $* *$ & 1,860 & 1,330 \\
\hline Venezuela & Maracay & 9022 & 3,63 & 11,4 & $* *$ & 0,588 & 0,415 \\
\hline U.S.A. & Weslaco & 9023 & 2,66 & 18,1 & $* *$ & 0,888 & 0,628 \\
\hline Guatemala & Las Vegas & 9025 & 5,48 & 6,7 & $* *$ & 0,674 & 0,477 \\
\hline El Salavador & Agroconsa & 9026 & 3,36 & 15,1 & wit & 0,979 & 0,692 \\
\hline
\end{tabular}


Cuadro 3. Medias de rendimiento e índices ambientales de 36 híbridos de maíz, del ensayo del PCCMCA 1990 evaluados en 20 ambientes.

\begin{tabular}{|c|c|c|c|c|c|c|c|c|c|c|c|c|c|c|c|c|c|c|c|c|c|}
\hline $\mathrm{HB}$ & 9001 & 9002 & 9003 & 9004 & 9025 & 9005 & 9006 & 9008 & 9007 & 9009 & 9010 & 9026 & 011 & 9013 & 9015 & 9016 & 9017 & 9020 & 9021 & 9023 & $x$ \\
\hline $\begin{array}{l}1 \\
2 \\
3 \\
4 \\
5 \\
6 \\
7 \\
8 \\
9\end{array}$ & $\begin{array}{l}3,22 \\
3,01 \\
2,72 \\
3,39 \\
3,54 \\
3,69 \\
3,97 \\
3,10 \\
2,89 \\
2,57 \\
3,54 \\
2,66 \\
3,00 \\
3,74 \\
3,07 \\
3,29 \\
2,83 \\
1,88 \\
3,35 \\
2,37 \\
3,49 \\
2,88 \\
3,67 \\
3,34 \\
3,28 \\
4,38 \\
3,73 \\
3,22 \\
3,92 \\
3,64 \\
2,62 \\
2,72 \\
3,18 \\
2,35 \\
3,39 \\
3,09\end{array}$ & $\begin{array}{l}3,72 \\
3,65 \\
3,35 \\
4,34 \\
3,19 \\
3,45 \\
3,83 \\
3,48 \\
3,38 \\
4,01 \\
3,97 \\
2,80 \\
4,44 \\
3,31 \\
3,12 \\
3,16 \\
3,73 \\
2,59 \\
4,16 \\
3,07 \\
3,78 \\
3,75 \\
3,55 \\
3,58 \\
3,27 \\
4,22 \\
3,87 \\
3,68 \\
4,16 \\
4,24 \\
3,20 \\
2,88 \\
2,84 \\
3,40 \\
3,54 \\
3,32\end{array}$ & $\begin{array}{l}5,49 \\
5,45 \\
4,95 \\
5,56 \\
5,22 \\
5,00 \\
5,06 \\
5,19 \\
5,20 \\
5,57 \\
5,61 \\
4,33 \\
5,39 \\
4,83 \\
6,11 \\
5,70 \\
5,37 \\
4,51 \\
4,94 \\
5,22 \\
5,33 \\
5,12 \\
5,61 \\
5,43 \\
4,17 \\
5,51 \\
5,71 \\
4,65 \\
5,92 \\
5,98 \\
4,50 \\
5,19 \\
4,63 \\
4,87 \\
5,43 \\
5,36\end{array}$ & $\begin{array}{l}8,66 \\
8,27 \\
8,33 \\
8,24 \\
8,25 \\
7,87 \\
7,94 \\
7,18 \\
6,59 \\
8,30 \\
8,68 \\
6,60 \\
8,13 \\
8,48 \\
9,33 \\
8,76 \\
8,43 \\
7,34 \\
8,74 \\
8,51 \\
8,59 \\
9,20 \\
8,62 \\
8,15 \\
7,51 \\
9,19 \\
8,56 \\
8,14 \\
9,09 \\
9,32 \\
7,38 \\
7,81 \\
7,77 \\
8,86 \\
8,92 \\
8,45\end{array}$ & $\begin{array}{l}6,10 \\
5,90 \\
5,46 \\
5,68 \\
5,47 \\
5,78 \\
5,48 \\
5,37 \\
5,04 \\
5,50 \\
5,52 \\
4,80 \\
5,16 \\
4,77 \\
5,46 \\
5,45 \\
5,62 \\
5,13 \\
6,21 \\
5,10 \\
5,92 \\
5,55 \\
5,92 \\
5,34 \\
4,07 \\
6,01 \\
5,56 \\
4,92 \\
5,79 \\
6,25 \\
4,72 \\
5,30 \\
5,13 \\
5,79 \\
5,93 \\
5,96\end{array}$ & $\begin{array}{l}3,54 \\
4,40 \\
4,03 \\
5,96 \\
5,00 \\
4,87 \\
4,87 \\
5,00 \\
4,12 \\
6,13 \\
5,12 \\
5,07 \\
4,96 \\
5,25 \\
3,77 \\
5,39 \\
4,92 \\
4,16 \\
4,96 \\
5,10 \\
5,20 \\
5,95 \\
4,38 \\
5,44 \\
4,19 \\
6,98 \\
5,80 \\
5,39 \\
5,05 \\
6,04 \\
2,98 \\
4,11 \\
5,09 \\
4,75 \\
4,56 \\
5,74\end{array}$ & $\begin{array}{l}7,09 \\
7,28 \\
6,71 \\
6,92 \\
6,97 \\
6,92 \\
6,58 \\
5,96 \\
4,98 \\
7,19 \\
7,44 \\
6,04 \\
6,70 \\
6,64 \\
8,24 \\
6,91 \\
6,67 \\
6,21 \\
6,45 \\
7,58 \\
7,34 \\
7,78 \\
6,95 \\
6,69 \\
5,84 \\
8,14 \\
6,50 \\
5,43 \\
6,20 \\
6,49 \\
5,69 \\
6,10 \\
7,07 \\
7,16 \\
7,81 \\
7,20\end{array}$ & $\begin{array}{l}4,70 \\
4,63 \\
4,32 \\
4,98 \\
3,24 \\
4,81 \\
3,88 \\
2,25 \\
3,61 \\
5,40 \\
4,27 \\
2,66 \\
3,65 \\
4,48 \\
4,62 \\
4,16 \\
5,06 \\
4,25 \\
4,75 \\
2,85 \\
3,89 \\
4,43 \\
4,24 \\
4,28 \\
3,23 \\
5,30 \\
4,35 \\
4,12 \\
4,76 \\
5,36 \\
3,25 \\
4,01 \\
4,04 \\
5,08 \\
5,76 \\
4,08\end{array}$ & $\begin{array}{l}5,51 \\
5,09 \\
4,07 \\
4,85 \\
5,95 \\
5,44 \\
4,79 \\
5,09 \\
5,32 \\
5,48 \\
6,64 \\
5,46 \\
5,45 \\
5,74 \\
5,18 \\
5,29 \\
6,19 \\
5,07 \\
5,26 \\
5,34 \\
6,66 \\
5,87 \\
6,24 \\
5,65 \\
4,83 \\
4,91 \\
5,77 \\
5,28 \\
6,28 \\
5,44 \\
4,24 \\
4,65 \\
4,71 \\
4,76\end{array}$ & $\begin{array}{l}5,34 \\
5,42 \\
4,90 \\
5,39 \\
5,04 \\
4,90 \\
5,10 \\
3,96 \\
4,77 \\
5,03 \\
5,58 \\
4,57 \\
4,83 \\
4,24 \\
5,94 \\
5,73 \\
6,03 \\
5,07 \\
5,11 \\
5,88 \\
6,11 \\
4,42 \\
6,28 \\
5,64 \\
4,36 \\
5,80 \\
5,17 \\
5,27 \\
5,37 \\
5,81 \\
4,82 \\
4,47 \\
5,14 \\
5,24 \\
5,52 \\
4,68\end{array}$ & $\begin{array}{l}5,02 \\
4,75 \\
3,85 \\
4,95 \\
4,69 \\
4,45 \\
4,64 \\
4,23 \\
4,28 \\
4,43 \\
4,72 \\
3,43 \\
4,91 \\
4,59 \\
5,34 \\
4,47 \\
5,16 \\
3,99 \\
4,73 \\
4,62 \\
5,17 \\
4,91 \\
5,78 \\
4,27 \\
3,88 \\
5,51 \\
5,11 \\
4,02 \\
5,28 \\
5,49 \\
4,14 \\
5,06 \\
4,43 \\
5,08 \\
5,27 \\
4,47\end{array}$ & $\begin{array}{l}2,97 \\
4,31 \\
3,54 \\
3,01 \\
3,38 \\
3,65 \\
2,97 \\
4,37 \\
3,36 \\
3,22 \\
3,26 \\
2,64 \\
3,85 \\
2,90 \\
2,92 \\
3,57 \\
3,65 \\
3,55 \\
3,27 \\
4,13 \\
3,36 \\
3,22 \\
3,58\end{array}$ & $\begin{array}{l}7,11 \\
6,93 \\
6,44 \\
7,59 \\
6,97 \\
6,07 \\
6,21 \\
6,32 \\
5,78 \\
5,59 \\
5,84 \\
5,30 \\
6,33 \\
6,78 \\
7,58 \\
5,76 \\
5,62 \\
5,59 \\
7,49 \\
6,67 \\
6,15 \\
6,16 \\
7,04 \\
7,50 \\
4,19 \\
6,46 \\
6,10 \\
5,42 \\
9,58 \\
6,87 \\
5\end{array}$ & $\begin{array}{l}8,81 \\
8,85 \\
7,78 \\
10,0 \\
8,20 \\
8,42 \\
8,12 \\
7,37 \\
6,16 \\
7,98 \\
8,17 \\
6,63 \\
8,37 \\
7,69 \\
9,56 \\
9,89 \\
8,44 \\
8,56 \\
8,53 \\
8,41 \\
8,23 \\
8,28 \\
10,5 \\
8,22 \\
7,61 \\
8,14 \\
8,43 \\
8,39 \\
8,92 \\
8,19 \\
6,05\end{array}$ & $\begin{array}{l}6,25 \\
5,76 \\
5,18 \\
6,25 \\
5,56 \\
5,, 61 \\
5,58 \\
5,30 \\
5,37 \\
6,07 \\
5,96 \\
5,34 \\
5,76 \\
4,96 \\
6,65 \\
6,10 \\
6,46 \\
5,17 \\
5,25 \\
6,18 \\
6,14 \\
6,16 \\
6,46 \\
5,20 \\
4,76 \\
7,19 \\
6,23 \\
5,58 \\
5,60 \\
7,11 \\
5,01 \\
5,42 \\
5,57 \\
5,50 \\
5,44 \\
5,55\end{array}$ & $\begin{array}{l}5,14 \\
4,77 \\
4,62 \\
5,69 \\
4,29 \\
4,52 \\
4,09 \\
4,58 \\
4,94 \\
5,20 \\
4,99 \\
4,08 \\
4,93 \\
4,92 \\
5,49 \\
5,09 \\
5,20 \\
4,48 \\
5,36 \\
5,89 \\
5,13 \\
4,80 \\
6,12 \\
4,85 \\
4,45 \\
5,48 \\
5,33 \\
4,89 \\
4,71 \\
5,04 \\
3,91 \\
4,62 \\
4,98 \\
4,72 \\
4,57 \\
4,65\end{array}$ & $\begin{array}{l}3,22 \\
4,32 \\
4,39 \\
4,63 \\
2,95 \\
3,09 \\
3,37 \\
2,75 \\
2,57 \\
4,85\end{array}$ & $\begin{array}{l}6,90 \\
6,34 \\
6,19 \\
6,45 \\
6,46 \\
6,63 \\
7,06 \\
5,72 \\
5,91 \\
6,53 \\
6,76 \\
4,66 \\
6,49 \\
5,56 \\
6,88 \\
6,60 \\
6,19 \\
4,85 \\
6,54 \\
5,97 \\
7,18 \\
5,62 \\
7,67 \\
6,08 \\
4,42 \\
6,93 \\
6,44 \\
5,28 \\
7,20 \\
7,69 \\
5,42 \\
6,07 \\
6,13\end{array}$ & $\begin{array}{l}7,40 \\
6,65 \\
5,59 \\
5,19 \\
7,13 \\
7,49 \\
6,38 \\
5,96 \\
5,87 \\
7,37 \\
7,43 \\
7,70 \\
7,37 \\
7,48 \\
8,19 \\
5,17 \\
6,90 \\
6,42 \\
6,55 \\
6,69 \\
7,21 \\
7,19 \\
6,67 \\
6,58 \\
7,70 \\
6,46 \\
8,37 \\
8,78 \\
7,12 \\
6,60\end{array}$ & $\begin{array}{l}2,31 \\
2,32 \\
2,20 \\
2,51 \\
2,23 \\
2,08 \\
3,75 \\
2,27 \\
2,78 \\
2,20 \\
1,90 \\
1,44 \\
3,36 \\
3,54 \\
2,87 \\
3,09 \\
3,08 \\
2,11 \\
2,15 \\
3,68 \\
3,05 \\
2,35 \\
3,22 \\
2,63 \\
2,65 \\
4,19 \\
3,67 \\
2,30 \\
2,04 \\
3,15 \\
3,01 \\
2,16 \\
2,30 \\
3,18 \\
3,06 \\
1,27\end{array}$ & $\begin{array}{l}5,46 \\
5,35 \\
4,95 \\
5,63 \\
5,17 \\
5,23 \\
5,18 \\
4,72 \\
4,55 \\
5,44 \\
5,40 \\
4,44 \\
5,33 \\
5,22 \\
5,77 \\
5,38 \\
5,42 \\
4,73 \\
5,48 \\
5,33 \\
5,62 \\
5,41 \\
5,80 \\
5,26 \\
5,49 \\
5,85 \\
5,53 \\
5,09 \\
5,67 \\
5,86 \\
4,44 \\
4,80 \\
5,02 \\
5,45 \\
5,70 \\
5,09\end{array}$ \\
\hline $1 \mathrm{~J}$ & & & $-0,03$ & & 0,22 & $\begin{array}{l}4,95 \\
-0,31\end{array}$ & & $-1,02$ & 0,07 & $-0,07$ & & $-1,90$ & & & & & & & & $\begin{array}{l}2,67 \\
-2,59\end{array}$ & , 26 \\
\hline
\end{tabular}

En el Cuadro 4, se incluye la información pertinente a los estimador es en el análisis de varianza combinado de 20 localidades, para variables de importancia económica y genética consideradas en el presente trabajo. La interacción híbrido-ambiente fue significativa para todas las variables, lo cual indica que los genotipos mostraron una respuesta relativamente diferente en algunas localidades. La variable de mayor importancia, mazorcas podridas mostró una variación de 4,00 a 13,00\% lo cual indica que existen genotipos resistentes a este factor adverso de origen biótico.

Cuadro 4. Estimadores en el análisis de varianza combinado para 36 híbridos evaluados en 20 localidades de Norte y Centro América, El Caribe y Venezuela. PCCMCA 1990.

\begin{tabular}{|c|c|c|c|c|c|c|}
\hline $\begin{array}{c}\text { Variable } \\
\text { Estadística }\end{array}$ & $\begin{array}{l}\text { Rendi- } \\
\text { miento }\end{array}$ & $\begin{array}{c}\text { Descu- } \\
\text { bierta } \\
(\%)\end{array}$ & $\begin{array}{l}\text { Podri- } \\
\text { da } \\
(\%)\end{array}$ & $\begin{array}{l}\text { Prolife- } \\
\text { racion } \\
(\%)\end{array}$ & $\begin{array}{l}\text { Altura } \\
(\mathrm{cm})\end{array}$ & $\begin{array}{l}\text { Días } \\
\text { Flor }\end{array}$ \\
\hline Media & 5,25 & 5,87 & 8,07 & 97,00 & 1,22 & 55 \\
\hline C.V. \% & 13,52 & 10,46 & 10,86 & 9,61 & 1,76 & 2,22 \\
\hline MDSH & 0,490 & 4,49 & 4,08 & 7,78 & 10,29 & 1,02 \\
\hline D.E.E. & 0,7032 & 0,92 & 0,87 & 9,32 & 2,14 & 1,22 \\
\hline Hibr.x amb. & $* x$ & $x *$ & $* *$ & $* *$ & $* *$ & tit \\
\hline Máximo & 5,85 & 16,00 & 12,41 & 1,09 & 1,43 & 58 \\
\hline Mínimo & 4,41 & 4,38 & 4,38 & 88 & 92 & 52 \\
\hline
\end{tabular}

Pudrición de mazorca fue la enfermedad de mayor importancia económica en Honduras, Costa Rica y Guatemala, considerándose pérdidas anuales de hasta el 20\%. Los híbrídos HA-46 y GB-35XGB-41 mostraron una considerable resistencia a esta enfermedad (4.41 vs 12.56 de H.56) este híbrido también superó significativamente en rendimiento al testigo H-5 (Cuadro 5). Cuadro 5. Medias de rendimiento y
características agronómicas de 36
híbridos de maíz evaluados en veinte
ambientes de Norte, Centro América
y El Caribe. PCCMCA, 1990.

\begin{tabular}{|c|c|c|c|c|c|c|c|}
\hline Híbridos & $\begin{array}{l}\text { Rend. } \\
\text { t/ha }\end{array}$ & $\begin{array}{l}\% \text { de } \\
\mathrm{H}-5\end{array}$ & $\mathrm{Df}$ & $\begin{array}{c}\text { A1tura } \\
(\mathrm{cm})\end{array}$ & $\begin{array}{c}\text { Descu- } \\
\text { bierta } \\
(\%)\end{array}$ & $\begin{array}{l}\text { Podri- } \\
\text { da } \\
(\%)\end{array}$ & $\begin{array}{c}\text { Proli- } \\
\text { ferac. } \\
(\%)\end{array}$ \\
\hline 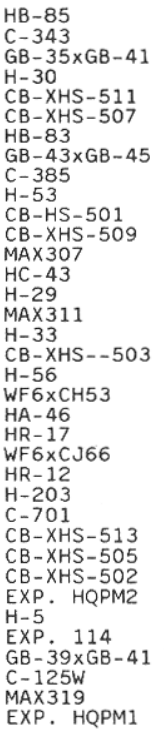 & $\begin{array}{l}5,85 \\
5,83 \\
5,81 \\
5,76 \\
5,68 \\
5,63 \\
5,61 \\
5,61 \\
5,53 \\
5,48 \\
5,47 \\
5,45 \\
5,43 \\
5,41 \\
5,40 \\
5,38 \\
5,38 \\
5,37 \\
5,34 \\
5,33 \\
5,26 \\
5,23 \\
5,23 \\
5,17 \\
5,17 \\
5,14 \\
5,09 \\
5,03 \\
5,01 \\
4,73 \\
4,72 \\
4,72 \\
4,56 \\
4,50 \\
4,43 \\
4,42\end{array}$ & $\begin{array}{l}124 \\
124 \\
123 \\
122 \\
120 \\
119 \\
119 \\
119 \\
117 \\
117 \\
117 \\
115 \\
115 \\
114 \\
114 \\
114 \\
114 \\
114 \\
113 \\
113 \\
111 \\
111 \\
111 \\
109 \\
109 \\
109 \\
109 \\
106 \\
106 \\
100 \\
100 \\
100 \\
97 \\
95 \\
94 \\
93\end{array}$ & $\begin{array}{l}54 \\
55 \\
55 \\
56 \\
55 \\
55 \\
56 \\
58 \\
56 \\
55 \\
55 \\
56 \\
56 \\
56 \\
57 \\
56 \\
57 \\
55 \\
58 \\
55 \\
56 \\
55 \\
57 \\
55 \\
55 \\
55 \\
55 \\
56 \\
55 \\
52 \\
58 \\
56 \\
56 \\
56 \\
55 \\
53\end{array}$ & $\begin{array}{l}120 \\
110 \\
116 \\
130 \\
122 \\
124 \\
119 \\
116 \\
111 \\
127 \\
140 \\
128 \\
127 \\
120 \\
132 \\
124 \\
131 \\
133 \\
144 \\
114 \\
112 \\
114 \\
128 \\
123 \\
123 \\
106 \\
134 \\
135 \\
127 \\
121 \\
137 \\
106 \\
102 \\
120 \\
92 \\
120\end{array}$ & $\begin{array}{l}8,06 \\
8,83 \\
6,44 \\
9,44 \\
8,94 \\
7,40 \\
7,47 \\
5,70 \\
13,32 \\
9,35 \\
7,35 \\
9,11 \\
16,70 \\
10,09 \\
8,19 \\
10,13 \\
7,31 \\
7,98 \\
9,95 \\
10,60 \\
5,25 \\
7,68 \\
8,66 \\
14,62 \\
12,34 \\
12,36 \\
8,93 \\
10,81 \\
9,53 \\
9,68 \\
4,50 \\
5,32 \\
5,12 \\
6,96 \\
4,44 \\
10,89\end{array}$ & $\begin{array}{l}7,21 \\
7,02 \\
4,41 \\
8,33 \\
6,89 \\
6,93 \\
6,45 \\
6,23 \\
9,87 \\
5,59 \\
9,71 \\
9,11 \\
7,27 \\
10,89 \\
7,95 \\
5,61 \\
8,65 \\
8,49 \\
12,33 \\
8,39 \\
5,52 \\
7,44 \\
7,36 \\
9,06 \\
7,77 \\
10,26 \\
9,63 \\
10,86 \\
7,31 \\
8,67 \\
8,49 \\
5,79 \\
7,44 \\
7,71 \\
11,70 \\
8,28\end{array}$ & $\begin{array}{l}97 \\
101 \\
96 \\
97 \\
93 \\
94 \\
96 \\
100 \\
100 \\
93 \\
96 \\
92 \\
97 \\
98 \\
96 \\
100 \\
101 \\
89 \\
93 \\
99 \\
109 \\
98 \\
99 \\
98 \\
100 \\
98 \\
89 \\
90 \\
98 \\
102 \\
92 \\
100 \\
105 \\
95 \\
90 \\
99\end{array}$ \\
\hline DMSH & 0,49 & & 1 & 10 & 4,49 & 4,08 & 8 \\
\hline
\end{tabular}


La prueba de Tukey aplicada al rendimiento descrimina en primer lugar 15 híbridos de los 36 evaluados en veinte localidades. El primer grupo lo constituyen HB-511, los cuales superaron al testigo H-5 (4.72 t/ha) hasta con 25\%, (Cuadro 5). El HB-85 y C-343, mostraron adaptación a la mayor ía de ambientes de prueba colocándose en los primeros cuatro lugares entre los 36 genotipos evaluados en veinte localidades (Cuadro $3)$.

El HA-46 mantiene su liderazgo en resistencia a pudrición de mazorca lo cual incrementa su potencial en el mercado regional y apoya el enfoque de mejoramiento para resistencia a factores adversos y bióticos que en el futuro contribuirán a una producción sostenida de maíz en la región. El segundo grupo los constituyen los híbridos C-385, H-53, XHS-509, H-29, XHS-501, MAX-307, HC43, esta serie de híbridos superó al testigo con rendimientos entre 0,490 a $0,800 \mathrm{t} / \mathrm{ha}$; entre ellos sobresalen C-385 y H-53 este último con resistencia al achaparramierito. Los híbridos CB-XHS-513, C-701, XHS-502, 505, 505, C-125W, MAX-319, EXPHQPM-1, HPQM-2 tuvieron un comportamiento similar al testigo H-5 (Cuadro 5). Los coeficientes de correlaciones simples estimados en el análisis combinado indican la magnitud de la asociación existente entre el rendimiento vs. mazorcas podridas, prolificidad y acame con coeficientes $\mathrm{r}=0,58^{* *}, 0,39^{*}$ y $0,59^{*}$, respectivamente (Cuadro 6).

Cuadro 6. Coeficientes de correlación simple entre rendimiento y características agronómicas. PCCMCA, 1990.

\begin{tabular}{lcccc}
\hline & \multicolumn{4}{c}{ MAZORCA } \\
\cline { 2 - 3 } Variables Rend. & Descubierta Podrida Proliferación Acame \\
\hline & & & & \\
Rend. & NS & $-0.53^{* *}$ & $0.30 * *$ & $-0.55^{* *}$ \\
M. Desc. & & NS & NS & NS \\
M. Pod. & & NS & $-0.58 * *$ \\
Prolif. & & & NS \\
Acame & & & \\
\hline
\end{tabular}

Las diferencias entre ambientes pueden cambiar con frecuencia la magnitud en el comportamiento de un genotipo a través de diferentes localidades de prueba. Los agricultores demandan nuevos cultivares que respondan consistentemente a la mayoría de condiciones ambientales; de aquí, la necesidad de desarrollar cultivares que interaccionen positivamente con el medio ambiente utilizando modelos que permitan la identificación de genotipos estables. El Cuadro 7, muestra las variabless de estabilidad del rendimiento. Los híbridos
HB- 85 y HB- 83 se mostraron estables $\left(\mathrm{Bi}=1, \mathrm{Sdi}^{2}=0\right)$ y llenan todas las caracter ísticas de una variedad deseable.

Cuadro 7. Medias y parámetros de estabilidad del rendimiento de 36 híbridos de maíz evaluados en 20 ambientes de Norte y Centro América, El Caribe y Venezuela. PCCMCA, 1990.

\begin{tabular}{|c|c|c|c|c|c|}
\hline Híbridos & $t / h a$ & $\begin{array}{l}\% \text { de } \\
\mathrm{H}-5\end{array}$ & $\mathrm{Bi}$ & Sdi2 & r \\
\hline $\mathrm{HB}-85$ & 5.85 & 124 & 0.980 & 0.070 & 0.96 \\
\hline$C-343$ & 5.85 & 124 & 0.920 & $0.110^{*}$ & 0.90 \\
\hline$G B-35 \times G B-41$ & 5.79 & 123 & $1.160^{\star}$ & 0.095 & 0.95 \\
\hline $\mathrm{H}-30$ & 5.76 & 122 & $1.280^{\star \star}$ & 0.084 & 0.96 \\
\hline $\mathrm{CB}-\mathrm{XHS}-511$ & 5.68 & 120 & 1. $140^{\star}$ & $0.100 *$ & 0.95 \\
\hline CB-XHS-507 & 5.63 & 119 & 1.070 & 0.086 & 0.95 \\
\hline $\mathrm{HB}-83$ & 5.61 & 119 & 1.020 & 0.060 & 0.97 \\
\hline$G B-43 \times G B-45$ & 5.61 & 119 & $1.180^{\star *}$ & $1.280^{* *}$ & 0.91 \\
\hline$c-385$ & 5.53 & 117 & 0.917 & 0.057 & 0.97 \\
\hline $\mathrm{H}-53$ & 5.48 & 117 & 1.000 & 0.086 & 0.95 \\
\hline CB-HS-501 & 5.47 & 117 & $1.150^{*}$ & 0.072 & 0.97 \\
\hline CB-XHS-509 & 5.44 & 115 & 1.100 & 0.092 & 0.95 \\
\hline MAX307 & 5.42 & 115 & 0.940 & 0.092 & 0.93 \\
\hline $\mathrm{HC}-43$ & 5.40 & 114 & 1.080 & 0.082 & 0.95 \\
\hline $\mathrm{H}-29$ & 5.40 & 114 & 0.954 & 0.068 & 0.96 \\
\hline $\operatorname{MAX} 311$ & 5.38 & 114 & 1.070 & 0.073 & 0.96 \\
\hline $\mathrm{H}-33$ & 5.37 & 114 & 1. $120^{*}$ & 0.072 & 0.97 \\
\hline CB-XHS --503 & 5.37 & 114 & 1.080 & 0.051 & 0.98 \\
\hline$H-56$ & 5.34 & 113 & 1.060 & 0.099 & 0.94 \\
\hline WF $6 \times \mathrm{CH} 53$ & 5.33 & 113 & 0.916 & 0.055 & 0.97 \\
\hline $\mathrm{HA}-46$ & 5.26 & 111 & 1.030 & 0.067 & 0.97 \\
\hline $\mathrm{HR}-17$ & 5.23 & 111 & 1.020 & 0.057 & 0.97 \\
\hline WF $6 \times \mathrm{CJ} 66$ & 5.22 & 111 & $0.850^{\star}$ & 0.077 & 0.94 \\
\hline HR- 12 & 5.17 & 109 & 1.100 & 0.063 & 0.97 \\
\hline $\mathrm{H}-203$ & 5.17 & 109 & $0.880^{*}$ & 0.071 & 0.95 \\
\hline$C-701$ & 5.14 & 109 & 0.900 & 0.078 & 0.94 \\
\hline CB-XHS -513 & 5.09 & 108 & 1.020 & $0.111^{*}$ & 0.91 \\
\hline CB-XHS-505 & 5.03 & 106 & 1.010 & 0.071 & 0.96 \\
\hline CB-XHS- 502 & 5.01 & 106 & 0.940 & 0.053 & 0.97 \\
\hline EXP. HQPM2 & 4.73 & 100 & 0.918 & 0.074 & 0.95 \\
\hline$H-5$ & 4.72 & 100 & 1.030 & 0.073 & 0.96 \\
\hline EXP. 114 & 4.72 & 100 & 0.720 ** & 0.130 * & 0.90 \\
\hline $\mathrm{GB}-39 \times \mathrm{GB}-41$ & 4.59 & 97 & $0.713^{* *}$ & 0.076 & 0.92 \\
\hline$C-125 \mathrm{~W}$ & 4.50 & 95 & $0.821 *$ & 0.081 & 0.93 \\
\hline MAX319 & 4.43 & 94 & $0.890^{*}$ & 0.083 & 0.93 \\
\hline EXP. QPM1 & 4.41 & 93 & $0.828^{\star}$ & 0.090 & 0.91 \\
\hline MDS & 0.49 & & & & \\
\hline
\end{tabular}

Los híbridos GB-35-XGB-41, H-30, CB-X-HS-511 presentaron los coeficientes de regresión más altos $(\mathrm{Bi}=$ $1,16^{*}, 1,28^{* *}$ y $1,14 *$ ) lo cual los clasifica como híbridos que responden en forma consistente a ambientes favorables, evidentemente estos híbridos tuvieron sus más altos rendimientos en los ambientes cuyo índice ambiental fue el más alto (Cuadro 3). Coeficientes de regresión altos a menudo están asociados a rendimientos altos, Córdova (1978).

Los coeficientes de regresión bajos y significativos a menudo están asociados con rendimientos bajos e identifican a cultivares que responden bien a ambientes pobres o subóptimos. En el presente trabajo cultivar es con coeficientes de regresión,(Bi) de 0,720, $0,713^{* *}, 0,821^{*}, 0,890^{*}$ y $0,828^{*}$ correspondieron a los híbridos con los más bajos rendimientos a través de los ambientes con índices negativos (Cuadro 7).

La respuesta diferencial de los cultivares evaluados en diferentes ambientes implica la utilización de metodologías que permitan discriminar adecuadamente 
los genotipos que contribuirán al progreso por selección. En un programa de mejoramiento, se necesita mucha precisión para seleccionar materiales superiores dentro de un grupo de genotipos cuyas diferencias en potencial de rendimiento son mínimas. Por otra parte, es necesario entender bien la interacción genotipo ambiente para poder hacer selecciones apropiadas para una región y evitar sorpresas desagradables en el futuro, Kempton (1984), Bradley et al. (1988), Gauch y Zobel (1988).

En el análisis combinado de estabilidad del rendimiento involucran 51 localidades y 204 repeticiones de los años 1988, 1989 y 1990 (Cuadro 8), los híbridos HB-85, HB-83 y H-30 rindieron 6,00, 5,73 y 5,72 t/ha, respectivamente superando al testigo $\mathrm{H}-5$ hasta con un $72 \%$.

Cuadro 8. Medias de rendimiento y parámetros de estabilidad del rendimiento de híbridos de maíz del PCCMCA evaluados en 20 ambientes. PCCMCA, 1988-1990.

\begin{tabular}{lllll}
\hline Híbridos & t/ha & $\begin{array}{l}\% \text { de } \\
\text { H-5 }\end{array}$ & Bi & Sdi2 \\
\hline HB-85 & 6.00 & 127 & 1.10 & 0.076 \\
HB-83 & 5.73 & 122 & 1.06 & 0.067 \\
H-30 & 5.72 & 122 & $1.22 * *$ & 0.088 \\
MAX-307 & 5.55 & 117 & 0.93 & $0.155^{*}$ \\
HA-46 & 5.53 & 117 & 1.07 & $0.160^{*}$ \\
XCH-53 & 5.34 & 113 & 1.01 & $0.230^{* *}$ \\
HR-17 & 5.22 & 110 & 1.12 & 0.050 \\
H-5 & 4.72 & 100 & 1.09 & 0.085 \\
MDSH & 0.403 & & & \\
\hline
\end{tabular}

Las diferencias entre ambiente pueden cambiar con frecuencia la magnitud en el comportamiento del genotipo a través de diferentes ambientes. Los agricultores demandan nuevos cultivares que respondan consistentemente la mayoría de condiciones ambientales. Este objetivo se puede lograr desarrollando genotipos que interaccionen positivamente con el medio ambiente utilizando modelos de estabilidad que capitalicen en interacción GxA, Zobel (1990). El programa de maíz de ICTA, Guatemala ha puesto énfasis especial en integrar al estabilidad del rendimiento y adaptación y sus proyectos de formación de híbridos, el progreso de este enfoque ha sido notable en el desarrollo de los híbridos HB-85 y HB83 que han mostrado notable estabilidad a través de años y ambientes. La acumulación de genes favorables que determinan la adaptación de los híbridos han sido notables ya que sus variables de estabilidad (bi $=1$, Sdi 2 $=0$ ) en rendimiento alto, los identifican como variables deseables, (Cuadro 8).
El análisis de varianza por el modelo AMMI (Cuadro 9), mostró alta significancia para todos los componentes involucrados en el análisis. La interacción GxA fue de gran importancia, esta captura el $28 \%$ de la SC para tratamientos.

Cuadro 9. Análisis de varianza del rendimiento para estimar los valores aditivos de genotipo, su interacción. Modelo AMMI. PCCMCA 1990.

\begin{tabular}{|c|c|c|c|c|}
\hline Fuente & $\mathrm{GL}$ & SC & CM & $\mathrm{Fa}=0,01$ \\
\hline Ambiente (A) & 50 & 3156,07 & 63,12 & $\star \star \star \star$ \\
\hline Genotipos (G) & 7 & 187,46 & 26,78 & $\star \star \star *$ \\
\hline$G \times A$ & 350 & 446,48 & 1,27 & $\star \star \star$ \\
\hline PCA 1 & 56 & 128,91 & 2,30 & $\star \star \star *$ \\
\hline Residual & 294 & 317,56 & 1,08 & $\star \star \star \star$ \\
\hline Error & 1071 & 602,06 & 0,56 & $\star \star \star *$ \\
\hline
\end{tabular}

El análisis de varianza para rendimiento mostró una interacción GxA significativa conteniendo el 10,53\% de la $\mathrm{SC}$ en que intervinieron tratamientos $(\mathrm{SCG}+\mathrm{SCA}+$ SCGXA).

El componente principal PCA1 contiene el 28,69\% de la SC de GxA. La Figura 1 muestra el comportamiento de los ambientes y genotipos conteniendo el 44,24 de la SC de tratamientos, Zobel et al. (1988) y Córdova (1989).

\section{RECONOCIMIENTO}

El presente documento es el resultado cooperativo de las siguientes entidades e insti-tuciones de Centroamérica, el Caribe y México; a quienes se agradece su invaluable aportación profesional: Costa Rica: Kenneth Jiménez, Carlos Salas (Universidad de Costa Rica). El Salvador: Adán Aguiluz, José Escobar (CENTA). Guatemala: Carlos Pérez, Nery Soto, José Luis Quemé, (ICTA); Antonio Cristiani, Héctor Deras, Jesús Merino (Cristiani Burcard); Roberto Velásquez (SEMINAL, TACSA). Honduras: Luis Brizuela, Víctor Méndez, Humberto Mejía, José Paz, (S.R.N.); Leonardo Corral El Zamorano). Nicaragua: Roger Urbina, Marvin Ovando (MIDINRA). México: Semillas TACSA, PIONEER Selecciones Genéticas). Alfonso Monteiro (CARGILL). Rep. Dominicana: Ramón Celado, Félix Navarro (CESDA). Panamá: Alfonso Alvarado, Daniel Pérez, Ismael Camargo (IDIAP). E.E.U.U.: Federico Poey (AGRIDEC) Hernán Cortez (PIONEER). Venezuela: Omar Rodríguez (PIONEER). 


\section{LITERATURA CITADA}

BLUM, A 1988. Plant breeding for stress environments. 18-28. CRC press.

BRADLEY, J.P.; KNITTLE K.H; TROYER AF. 1988. Statistical methods in seed corn product selection. J. Prod. Agri. 1:34-38.

CARBALlO, C. A.; MARQUEZ, S.F. 1970. Comparación de variedades de maíz de El Bajio y La Mesa Central por su rendimiento y estabilidad. Agro-Ciencias 5(1): 129-146.

CORDOVA, H.S. 1978. Uso de parámetros de estabilidad para evaluar el comportamiento de variedades. Guatemala, ICfA. 35 p.

CORDOV A, H. S.; RAUN, W.; BARKER, T. 1988. El uso de parámetros de estabilidad para determinar la adaptación de 36 cultivares de maíz Simposiun Modelos de Estabilidad para Evaluar la Adaptación de Cultivos. XXXIV Reunión Anual del PCCMCA San José, Costa Rica. 5p.

CORDOVA, H.S. 1989. Evaluación de 36 cultivares de maíz en 20 ambientes de Centroamérica, Panamá y El Caribe PCCMCA 1988. XXXV Reunión Anual del PCCMCA San Pedro Sula, Honduras. abril 2 al 7.vol 3. 903p.

DAVILA, FA; CORDOVA, II.S; POEY, F.R.1978. Uso de parámetros de estabilidad en la evaluación de variedades comerciales y experimentales de maíz Zea mays L.ñ (1) Zona Media. XXIV Reunión Anual del PCCMCA. San Salvador.El Salvador. p.M31/1-
EBERHART, SA; RUSSELL, W A 1\%6. Stability parameters for comparing varieties. Crop. Sci. 6:36-40.

FINIA Y, K.. W.; WILKINSON G. N. 1963. The analysis of adaptation in plant breeding programe, Aus. J. of Agriculture research, 14, 742.

GAUCH, H. G.; ZOBEL. R. W. 1988. Predictive and postdictive success of statistical analysis of yield trials. Theor. App!. Genet. 76:1 10 .

GAUCH, KG.; ZOBEL, R. W. 1989. Accuracy and selection success in yield trial analysis. Theor. App!. Genet. 77:473-481.

KANG, M. S. 1990. Genotype by environment interaction and its implication in plant breeding. Symposium on: Genotypes by environment interaction and plant breeding. Ed. by Manjit, S. Kang, LSU, Agricultural Center.

KEMPTON, R. A 1984. The use of biplots in interpreting variety by environment interactions. J. A.gric. Sci. 103:123-136.

ZOBEL, R. W.; WRIGTHT, MJ.; GAUCH, H.G. 1988. Statistical analysis of a yield trial. Agron. J. 80:388-393.

ZOBEL, R. W. 1990. A powerful statistical model for understanding genotype-by-environmental interaction in genotype-by-environmental interaction and plant breeding. L.S.U. Agricultural center. 